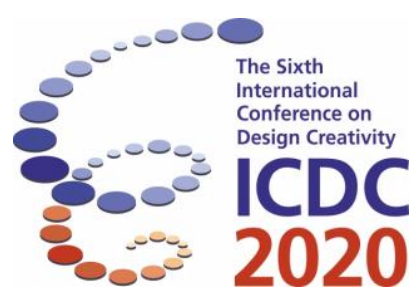

\title{
An analysis of socio-cognitive activities during co- creative design supported by spatialized augmented reality
}

\author{
Cédric Masclet, Laetitia Baldacchino and Jean-François Boujut \\ Univ. Grenoble Alpes, CNRS, Grenoble INP*, G-SCOP, 38000 Grenoble, France \\ *Institute of Engineering Univ. Grenoble Alpes
}

\begin{abstract}
This study investigates the mechanisms by which spatial augmented reality (SAR) technology can affect socio-cognitive processes in groups involved in co-creative design sessions. A protocol analysis has been conducted to investigate three different design sessions involving experienced designers and end-users on a product design task: a design session supported by classic design representations (usual design practices), a design session supported by non-spatial augmented reality (AR), and a session supported by SAR technology. While results don't clearly show that SAR or AR technology increase end-user/client commitment, they illustrate the ability for these technologies to allow browsing through more ideas during a co-creative design sessions. Furthermore, it tends to reduce time spent on ideas, compared to a traditional session. We also noted that the introduction of these different technologies does not modify the profiles of the sessions in terms of cognitive activities. This tends to demonstrate that the technology itself does not impair the design activity.
\end{abstract}

Keywords: Co-creativity, Spatial Augmented Reality, cognitive activities, interaction analysis

\section{Introduction}

Co-creative design aims at increasing originality and relevance of the products and shorten product design cycles. These approach refers to involving end-users in the design process in order to ensure the relevance of the selected solutions. A co-creative design session allows designers to collect from end-users both feedback on pre-existing solutions and new ideas (i.e. design proposals). Therefore, this type of design session is characterized by both divergent processes (idea generation) and convergent processes (idea evaluation and selection); in this paper, any proposition of design solution will be designated by the term 'idea'.

However, while designers are design experts, end-users have, in principle, no design training. This expertise gap as well as the diversity of backgrounds can lead to communication barriers which affect session productivity especially in the manipulation of design representations. A solution to overcome this limitation is to introduce tools which facilitate co-creative design sessions.

This study is based on a technology developed within the framework of the SPARK H2020 European research project. The Spatial Augmented Reality (SAR) consists in projecting graphic elements such as colors, textures, finishing or logos on physical objects in order to simulate the final visual aspect of 
the product. The physical object adorned with the graphic information is referred to as "mixed object" because it has both real and virtual part. A tracking device allows to maintain the graphic information on the object when it is handled. Graphic elements projected on the tangible part of the artefact can be modified in real time thanks to a dedicated interface made available for Designers only.

As Bonnardel et Zenasni (2010) argue, the development and the assessment of new technologies aiming to support creative design should rely on a deep understanding of designers' cognitive processes, and more particularly, an understanding of designers' cognitive processes while using these technologies. The intent is that the tools being developed are adapted to the participants' cognitive processes, instead of requiring them to adapt to the tools.

Therefore, the aim of this study is to determine if the SAR technology affects the cognitive processes of a co-creative design session, and if so in which manner.

\section{Representations in collaborative design activities}

Because they are complex, design problems usually require knowledge distributed among actors who have different perspectives and backgrounds. Indeed, "When we look at actual human practices, we see that human cognition aspires to efficiency in distributing intelligence - across individuals, environment, external symbolic representations, tools, and artifacts - as a means of coping with the complexity of activities we often call mental" (Salomon, 1993a). In a 1994 study, Rogers et Ellis(1994) propose the framework of Distributed Cognition for analysing and explaining collaborative working. They define the focus of the analysis as the relations and interactions between the individuals and the artefacts. Therefore, to study cognitive processes in real design sessions it seems relevant to study design as a collaborative practice and to focus on the interaction between individuals as well as between individuals and artefacts.

De Vries et al. characterize the role of representations in design using Duval's three cognitive functions of representations in mathematics: objectification, communication and computation (de Vries \& Masclet 2012). These functions are described at the individual level but can be extended to group cognition. We are interested in the first two functions. Objectification describes how representations can make abstract ideas perceivable by the senses, in other words the representation allows the individual to become "aware of something through expression for oneself". This is also true at the group level when "an artifact gradually develops from the ideas of the members of a design team". The second cognitive function of representation is communication because they allow "exchanging information between team members with expertise in different domains". These have to be approximated to the concept of "externalization" described by Bonnardel \& Zenasnial (2010) as the process of "creation and modification of external representations of the object to be designed, such as drawings, sketches or 3D virtual representations produced with a CAD system". This process facilitates communication between designers and other stakeholders and supports the development of a shared understanding.

(Boujut \& Blanco, 2003) placed external representations at the center of interactions by naming them "intermediary objects" to indicate that they provide a common referential which supports communication between multiple actors and between successive work stages.

(Goldschmidt, 2007) studied the role of visual representations in building shared mental models in design teams. She explains that task mental models in design have the specificity of being contingent on visual representations. To design a tangible entity, hypothetical solutions are generated and manipulated while actors discuss about them.

Christensen \& Schunn (2009) examined the role of different types of external representations in design and pointed out the superior perceptual read-off of prototypes over sketches because they provide better support in more sense modalities (haptic, gustatory, olfactory and auditory).

Therefore, it would be useful to better understand how representations providing different perceptual read-offs affect the cognitive processes taking place during the completion of the design task.

\section{Research question}

The technical set of a co-creative session provides the participants with different types of representations. Characterizing these external representations allow us to articulate expectations 
concerning stakeholders' added value to the co-creative session. This leads us to frame the following research question: when used in real co-creative design sessions, does a SAR system facilitate the unfolding of the collective creative process, compared to sessions occurring in standard settings?

To answer this question, we studied three types of co-creative design sessions:

- A standard session (referred to as STD). The participants are provided with a screen to display designers' proposals prepared before the session (mostly slides with static CAD representations and charts of solutions), existing physical prototypes and a Pantone ${ }^{\circledR}$.

- An augmented reality (referred to as AR) session. Interaction with the artefact is digitally mediated by a tablet embedding a see-through technology which is preventing a direct visualisation of the product. This technology allows real-time modifications thanks to a software interface manipulated by the designers to change visual elements projected over the see-through apparatus.

- A Spatial Augmented Reality (referred to as SAR) session. Graphic information is directly projected on a tangible artefact. There are no helmet, glasses or intermediary screen needed and the tracking device enables direct manipulation of the artefact. The interaction is unmediated and intended to provide a certain realism of interaction. Participants can visualize and manipulate the design object as naturally as possible.

Unlike in the STD condition, SAR and AR technology enable real-time evolution of the visual aspect of the design object by allowing real-time modification of the digital information. They allow the generation of external representations of the ideas generated by the participants during the session, not exclusively the representations generated by the designers prior to the session.

We assume that being closer to the real situation of use of the product makes the representation more accessible to participants with no design expertise. It should facilitate expression and evaluation of ideas or opinions. This characterisation of the external representations provided by the different tools, leads us to formulate several hypotheses. First, we expect the proportion of End-user global participation, compared to Designer participation, to be larger in SAR condition than in other conditions. Secondly, we expect the number of ideas discussed throughout the session to be higher in SAR condition than in AR condition and STD condition. Finaly we expect discussion time spent on each idea to be shorter in SAR condition than in AR condition than in STD condition.

\section{Methodology}

\subsection{Protocol analysis}

Protocol analysis (Ericsson \& Simon, 1993) has been extensively used and has been considered to be the most appropriate empirical research method to study designers' cognitive abilities. Design protocols are recordings of subjects' verbal behavior while performing a design task. Because design activity is considered to involve information processing, which is not only symbolic but also visual, the object of interest of design protocols can be the verbal discourse, but also non-verbal elements such as gestures or the production of sketches. Whatever the goal of protocol studies is, the methodological process is the following: after the definition of a coding scheme to characterize what happens during the design sessions, recording and transcription of the designers' verbal interactions, segmentation of the transcription, classification of segments according to the coding scheme. The sequences of codes obtained can be analysed (Gero \& McNeill, 1998).

We chose to study the content of verbal interactions which provide information about the group's cognitive processes. Using existing coding schemes from the literature we built a coding scheme adapted to our research question. Because we wanted to capture the convergent and divergent creative processes of co-creative design sessions as well as cognitive activities typical of design tasks we used:

- A coding scheme which accounts for the creative process by capturing the evolution of ideas throughout the session (Harvey, 2013).

- A coding scheme, named COMET, which accounts for cognitive activities related to collaborative design (Darses et al., 2001).

A first coding scheme, based on what Harvey and Kou defined as the aggregation of intermediary codes, fall into three categories: idea generation, idea evaluation and idea decision. This code applies 
to idea related conversation and aims at characterizing the idea-related verbal units. After testing the coding schemes on video extracts, we wanted to improve these intermediary codes to make them more specific to a product design task, and thus facilitate the coding. Therefore, we redefined the intermediary codes using the second coding scheme which was designed to analyze collective design processes (Darses et al., 2001). We obtained the following grid (Table 1).

We would also like to emphasize on the coding of the category Decide. Indeed, in this type of session decisions are relatively difficult to identify. In a study investigating argumentative processes in collaborative design, Darses et al (2006) explains that, often put on hold pending further information to be obtained, decisions are usually reviewed and modified at subsequent meetings and that explicit agreement and disagreement are rare. This is particularly true in this typical situations of co-design, where the outputs of the sessions are not explicit decisions. The objective of the designers is to collect insight from end-users and the outputs are promising ideas that the designers will bring further in the design process, beyond the session. they will sometimes explicitly express the fact that they want to store a certain idea for later. Designers and end-users will often reach an agreement about accepting or rejecting an idea without a decision being explicitely spelled out. We chose to collect both explicit decisions (DEC) as well as agreements. We based our definition on Harvey et al.'s coding scheme which defined decision related units as "explicit consensus of agreement or disagreement with an idea or an expressed decision by one or more group members that was not challenged". Therefore, it is difficult to capture decisions in an objective and exhaustive manner. However our choices concerning the coding of Decisions allow us to capture relevant information concerning agreements and decisions throughout the session. Our coding scheme does not collect decisions which derive from other decisions. For example, if different colors are discussed and the participants agree that yellow is a lead to be pursued, the coder will not infer from that that other colors are rejected.

Finally, we assessed observer reliability on a seven-minute session extract by calculating the Cohen's Kappa (Cohen, 1960) over a double-coding which concluded on a high level of reliability $(\kappa=0,8)$.

Table 1 - Coding scheme

\begin{tabular}{|l|l|}
\hline \multicolumn{1}{|c|}{$\begin{array}{c}\text { Idea Related Coding } \\
\text { categories }\end{array}$} & \multicolumn{1}{c|}{ Codes of cognitive activities } \\
\hline Idea introduction related & $\begin{array}{l}\text { PRES (Presentation) / GEN (Generation) / CLAR (Clarification: } \\
\text { noncritical discussion of an idea that aims at understanding the idea)/ } \\
\text { REINT (Reintroduction) }\end{array}$ \\
\hline Idea evaluation related & $\begin{array}{l}\text { EVAL (Evaluation) / INT (Interpretation) / JUST (Justification) / INFO } \\
\text { (Information exchange about the problem framework) / AG/DISAG } \\
\text { (Agreement/Disagreement) }\end{array}$ \\
\hline Idea decision related & DEC (Explicit decision-making) /AG (Consensual agreement) \\
\hline
\end{tabular}

\subsection{Observations}

The observations were carried out in partnership with a product design and innovation agency named Stimulo. In order to limit variations, same designers (from Stimulo) led the three sessions and the design issues discussed were similar from one session to another. Three different End-users were enrolled for each session. Designers has been trained to the SAR technology before the observations, providing sufficient experience to deal with SAR and AR (wich used the same GUI). Real design briefs of real products were comparable upon the three conditions (STD/AR/SAR), consisting in defining colors and finishes of three hand-held size real product under development. External representations where respectively an existing prototype with Pantone ${ }^{\circledR}$ and a TV screen for STD condition, a tablet implementing AR technology with an existing prototype for AR condition, and the SAR system for the SAR condition. 


\begin{tabular}{|l|l|}
\hline STD condition & AR condition \\
\hline aviuno
\end{tabular}

Figure 1: pictures of physical prototypes of the products discussed during the co-design sessions.

\section{Results}

Preliminary results show that the distribution of idea-related (IR) speech among activities seems to be the same for all three conditions. Evaluation ranges from 52 to $64 \%$ while decide slightly oscillates between 9 and $14 \%$ of the total IR speech. Therefore, we can state that the introduction of AR and SAR technology into co-creative sessions does not significantly modify the proportions of cognitive activities, compared to a session taking place in a traditional setting.

\subsection{End-user commitment}

Concerning actor commitment during a co-creative design session, we saw that designers occupy the larger proportion of spoken time in all three conditions: 64\% in STD condition and 76\% in both SAR and AR condition.

Designers occupy the largest proportion of idea-related (IR) speech time between Designers and Endusers, for all three conditions. However, the proportions are more balanced than for spoken time: Designers hold 56\% of IR speech time in STD condition, 60\% in SAR condition and 71\% in AR condition.

Then, we focused on the distribution of IR speech among the more specific cognitive activities. In the SAR condition, End-users occupy $65 \%$ of time spent on idea generation (GEN) while they occupy only $36 \%$ and $27 \%$ respectively in STD and AR condition.

\subsection{Exploration of the solution space}

The SAR session allowed participants to scan through more ideas than the AR or STD conditions. Observations are in line with our hypothesis can't be only explained by a longer session duration (Table 2). However, we note that the number of ideas for which discussion led to a decision remains similar in all three conditions.

Table 2 - Number of ideas discussed and number of ideas decided on per session in each condition

\begin{tabular}{|c|c|c|c|}
\hline Session (duration) & STD (40') & AR(35') & SAR(45') \\
\hline $\begin{array}{c}\text { Number of ideas discussed/decided per } \\
\text { session }\end{array}$ & $30 / 17$ & $32 / 22$ & $40 / 18$ \\
\hline
\end{tabular}

We formulated an hypothesis according to which discussion time spent on each idea would be shorter in SAR condition than in AR condition than in STD condition. Figure 2 represents the number of ideas discussed per interval of discussion time spent on an idea for each condition. 


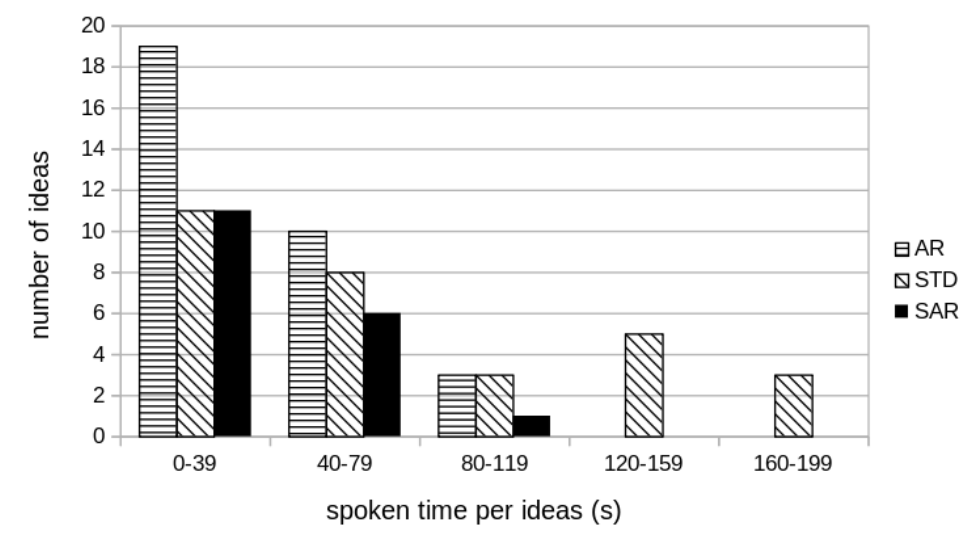

Figure 2. Spoken time spent on ideas

In the SAR and AR conditions, more ideas are discussed in a shorter amount of time, while in the STD condition there are more ideas on which more discussion time is spent. For example, in the STD condition there are 8 ideas for which more than 119 seconds of discussion time was spent, while in the SAR and AR conditions none of the ideas took more than 119 seconds. This contrast seems to be a distinction point between the STD session and XAR sessions.

\section{Discussion and future work}

\subsection{Discussion}

When comparing conditions, SAR and AR technologies don't seem to lead to more end-user commitment. Designers still occupy a larger part of verbal exchanges than end-users in all three conditions. This seems to reflect the fact that co-design sessions are structured and led by the designers. Our hypothesis concerning the ease for end-user to communicate and suggest modification is not clearly supported by our observations. This is also true when comparing speaking time for sole idea-related speech, but the difference is reduced. This is coherent since non idea-related (NIR) speech is mainly about the design process, recalling the history of the project, tool management and designers' asides. It was expected that end-users would be more involved in the IR conversation. Although we are witnessing a slight increase of participation for end-user in SAR and AR technologies, we cannot consider it as a significant turnover.

We also noted that end-users spent a larger proportion of time in generating ideas in the SAR session. This is a crucial point since generating ideas (which designers would not have thought of themselves) is one of the objectives of co-creative design sessions.

Concerning the distribution of activities, the three conditions appear similar in terms of time spent, despite the differences in design settings, in the product discussed during the sessions, and in end-user profiles. Therefore, it seems that the AR and SAR tools do not transform the socio-cognitive processes taking place during a session supported by traditional design representations.

We would like to underline that the results obtained must be interpreted cautiously because end-user participation may be strongly impacted by other factors, notably end-user personalities and the type of design issues discussed through the session. The style of management of the session could also be a factor, however since we ensured that the same designer managed all three sessions, its influence should be limited. We also think that bringing new technologies without familiarizing the end-users beforehand may impact their commitment negatively. Indeed, the presence of an unfamiliar technology could divert the end-users' attention from the design task, either because the tool itself is attractive or because its unfamiliar aspects are destabilizing. For example, one of the end-users in the AR session seemed disturbed by the color differences between the tablet for modifying the graphics and the tablet dedicated to the visualization of the prototype. His participation were infrequent and often related to this issue rather than the design task itself. The importance of familiarizing subjects is underlined by Bonnardel et al. "although it may seem attractive to use a new technology for 
performing tasks which would be difficult or impossible with classical technologies, the use of new technologies may require a period of familiarization before allowing users to benefit from the new functionalities offered by such systems" (Bonnardel \& Zenasni, 2010).

Another assumption is that, in SAR and AR conditions, the externalization of ideas generated during the session thanks to real time modification of the design representation would facilitate their evaluation. Participants would be more efficient in either reaching an agreement or proposing an alternative. We hypothesized that SAR and AR technologies would allow to reduce the time spent discussing ideas and that consequently participants would browse through more ideas during a session. The results seem to support this hypothesis, since in the SAR and the AR conditions more ideas were browsed through than in the SAR condition. Furthermore, in the SAR and AR conditions, more ideas are discussed in a shorter amount of time. However, there is little difference in the number of ideas decided-on over the whole session. In further work it would be interesting to reconsider the assumption that browsing through more ideas is useful for the productivity of the session.

During the session in STD condition, as the participants were discussing color associations for the product, one of the end-users said "It's hard to imagine all three colors together if you don't have the picture". It seems that it was challenging for this participant to express a preference for a color without visualizing the rendering on the product. This type of statement illustrates how having external representations of ideas could facilitate evaluation.

We observe that the exchanges are dominated by idea evaluation related discussion. This is not surprising since we built our coding scheme to account for this activity which is considered central in both divergent and convergent dimensions of design and creative tasks (Harvey, 2013). This was particularly appropriate for co-creative design sessions because their objective is to collect end-users' feedback to eliminate irrelevant propositions and collect new relevant ideas which the designers would not have thought of themselves. The large amount of time spent on the activities described by the category "Evaluate" also accounts for the fact that we chose to include in this category the activity of information exchange about the problem framework (INFO). We considered that this activity was related to idea evaluation because it is information exchange that serves evaluation, which allows participants to evaluate ideas appropriately.

\subsection{Future work}

We noticed that information exchange about the problem framework (INFO) represented the largest proportions of cognitive activities in all three conditions. The fact that our coding scheme which is focused on the generation of solutions reports back such time-use seems to illustrate the co-evolution of problem and solution thanks to iterations between problem space and solution space (Kolodner \& Wills,1996) (Cross, 2001). It could be useful to further separate INFO into finer codes to differentiate information exchanges which directly concerns the product from information exchanges which refers to context and simulation of use. This would allow us to see the proportions of these types of information exchange as well as the artefacts they are supported by.

The significance of our results is limited by the fact that we only analyzed one session per condition with the same designers team. It would be useful to validate or invalidate the insights from this study with further research based on a larger number of sessions led by different designers working on different products. Introducing the technology to the end-users before the sessions could impact positively their commitment by preventing the cautiousness we observed during SAR and AR sessions. Finally, future work could investigate the sequencing of cognitive activities with a Lag Sequential Analysis (LSA) to identify differences and similarities between the three conditions. This method should help to determine whether the frequency of a given code is dependent of the frequency of another one (d'Astous et al., 2004) or not.

\section{Conclusion}

The purpose of the paper is to investigate the ways in which spatial augmented reality (SAR) technology can affect socio-cognitive processes in groups involved in co-creative design sessions. We hypothesize that the real time changeable external design representations provided by the technology 
facilitates both communication and idea evaluation thus increasing end-user commitment as well as the effectiveness of the exploration of the solution space.

The conducted analysis of both end-user commitment and the distribution of cognitive activities throughout each session shows that despite the introduction of new technologies (AR or SAR), the profiles of the sessions remain consistent. This tends to demonstrate that the technology does not impair or modify the design activities.

\section{Acknowledgment}

The work reported in this paper is part of the SPARK project, funded by the European Union's Horizon 2020 research and innovation programme under grant agreement No.688417. This paper reflects only the authors' views and the European Commission is not responsible for any use that may be made of the information it contains. The authors would like to thank all the partners of the SPARK Consortium and especially Maud Poulin and Céline Gros for their extensive support and valuable contribution to the development of this research.

\section{References}

Bonnardel, N., \& Zenasni, F. (2010). The Impact of Technology on Creativity in Design : An Enhancement? Creativity and Innovation Management 19(2).

Boujut, J.-F., \& Blanco, E. (2003). Intermediary objects as a means to foster co-operation in engineering design. Computer Supported Cooperative Work (CSCW), 205-219.

Christensen, B., \& Schunn, C. (2009). The Role and Impact of Mental Simulation in Design S F. Appl. Cognit. Psychol. 23, 327-344.

Cohen, J. (1960). A coefficient of agreement for nominal scales. Educational and Psychosocial Measurement, 20, 37-46.

Cross, N. (2001). Chapter 5 - Design Cognition: Results from Protocol and other Empirical Studies of Design Activity. Dans C. Eastman, W. McCracken, \& W. Newstetter, Design Knowing and Learning: Cognition in Design Education (pp. 79-103).

Darses, F. (2006). Analyse du processus d'argumentation dans une situation de reconception collective d'outillages. Le Travail Humain (69), 317-347.

Darses, F., Détienne, F., Falzon, P., \& Visser, W. (2001). COMET. A Method for Analysing. RR-4258, INRIA.

d'Astous, P., Détienne, F., Visser, W., \& Robillard, P. (2004). Changing our view on design evaluation meetings methodology: a study of software technical review meetings. Design Studies.

de Vries, E., \& Masclet, C. (2012). A framework for the study of external representations in collaborative design settings. International Journal of Human-Computer Studies.

Ericsson, K., \& Simon, H. (1993). Protocol Analysis : Verbal Reports as Data (Rev. ed.). Cambridge, MA, US: MIT Press.

Gero, J., \& McNeill, T. (1998). Changing our view on design evaluation meetings methodology: a study of software technical review meetings. Design Studies, Vol. 19 No.1, 21-61.

Goldschmidt, G. (2007). To see eye to eye: The role of visual representations in building shared mental models in design teams. CoDesign, 3, 43-50. .

Harvey, S. \&. (2013). Collective engagement in creative tasks: The role of evaluation in the creative process in groups. Administrative Science Quarterly, 58, 346-386.

Kolodner, J., \& Wills, L. (1996). Powers of observation in creative design. . Design Studies 17(4), 385-416.

Rogers, Y., \& Ellis, J. (1994). Distributed cognition: an alternative framework for analysing and explaining collaborative working. Journal of Information Technology, 9(2), 119-128.

Salomon, G. (1993a). Distributed cognitions: Psychological and educational considerations. New York: Cambridge University Press. 